\title{
Determination of the Temperature Dependent Thermal Expansion Coefficients of Bulk AlN by HRXRD
}

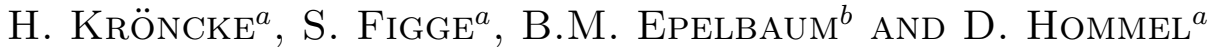 \\ ${ }^{a}$ Institute of Solid State Physics, Section Semiconductor Epitaxy \\ University of Bremen, Otto-Hahn-Allee 1, D-28359 Bremen, Germany \\ ${ }^{b}$ Department of Materials Science 6, University of Erlangen-Nürnberg \\ Martensstraße 7, D-91058 Erlangen, Germany
}

We measured the lattice constants of bulk aluminum nitride crystals at various temperatures by high resolution X-ray diffraction. By the use of a high temperature chamber and a X-ray cryostat a temperature regime from 20 to $1210 \mathrm{~K}$ was available. Furthermore, the measured data were fitted by Einstein- and Debye models which yield reliable parameters for the calculation of the thermal expansion coefficients of AlN.

PACS numbers: 01.30.Cc, 61.05.cp, 81.05.Ea, 65.40.De

\section{Introduction}

Aluminum nitride is a promising substrate material due to its special characteristics. Because of its large band gap of $6.2 \mathrm{eV}$, AlN could be used for the realization of optoelectronic devices with an emission in the near to mid-UV region. Possible usages are the purification of water or data storage. Furthermore, AlN is an insulator and has a high thermal conductivity, so it can be used as a substrate for high power and high frequency devices. The hardness also results in high sound velocity, which is useful for the construction of surface acoustic wave (SAW) devices, which are used in communication devices or as sensors. A realization of all these applications fails due to lack of substrates of sufficient size and quality. Because of the challenging production, which is only possible from vapor phase, up to now only few companies offer substrates in a reasonable quality. For the production of (opto-)electronic devices, usually epitaxial methods are used, which operate at high temperatures. A big problem is the bending and cracking of the deposited layers, caused by the different lattice constants of the used materials, which lead to an intrinsic stress. Furthermore, the thermal expansion can cause additional stress which has to be considered. Hence it is of technological interest to know the thermal behavior of AlN, which will play an increasing role in optoelectronics. 


\section{Experimental}

All the samples investigated in this study were grown by physical vapor transport (PVT) at the University of Erlangen using a tungsten crucible under nitrogen atmosphere at pressure below 1000 mbar in a two-step growth mode. In the first growth run commercial AlN powder, which contains up to one weight percent of $\mathrm{Al}_{2} \mathrm{O}_{3}$ due to its large surface, is heated and condensed again into distinguishable AlN and $\mathrm{Al}_{3} \mathrm{O}_{3} \mathrm{~N}$ layers. The $\mathrm{Al}_{3} \mathrm{O}_{3} \mathrm{~N}$ is then removed mechanically. In the consequent run AlN crystals nucleate at temperatures up to $2250^{\circ} \mathrm{C}$, while the source material is kept $50-75^{\circ} \mathrm{C}$ above. On the one hand, a spontaneous nucleation of AlN at the crucible wall leads to single crystals (Fig. 1), which differ in size and shape by the growth temperature. On the other hand, the nucleation at the top of the crucible leads to polycrystalline AlN or multi-grain material (Fig. 2), which also has a very low oxygen content of $1 \times 10^{19} \mathrm{~cm}^{-3}$. Further details on the growth can be found in [1].

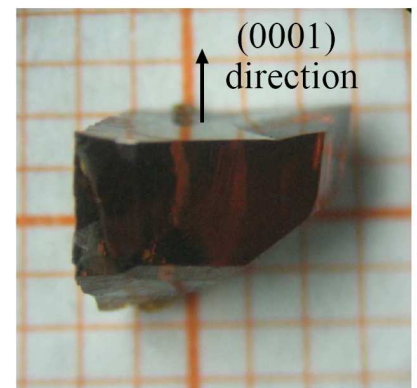

Fig. 1

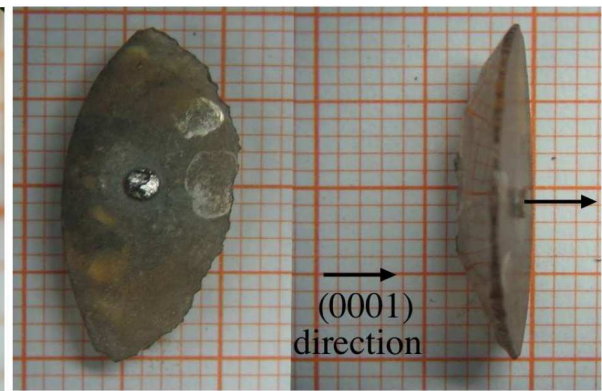

Fig. 2

Fig. 1. Single crystal.

Fig. 2. Multi-grain material.

All measurements were performed utilizing a Phillips X-Pert MRD high resolution diffractometer with a $\mathrm{Cu} K_{\alpha}$ cathode $(\lambda=1.540553 \AA)$ equipped with a twofold hybrid monochromator and a threefold $\mathrm{Ge}(220)$ analyzer.

Because the major growth rate of AlN is along the $c$-direction, all samples exhibit distinguished surfaces in this direction. From this follows that for a proper mounting the $c$-plane has to be used, so that the $c$-lattice constant could be determined directly from the symmetric reflexes 002, 004, 006. In contrast, the $a$-lattice constant had to be calculated from the asymmetric reflexes 103, 105, 205, using the $c$-lattice constant determined before. Furthermore, some corrections are necessary, to calculate the lattice plane distance from the Bragg angle $(\theta)$. Especially, the difference in the refractive index $(\delta=1-n)$ and a tilt of the lattice planes to the surface $(\alpha)$ has to be considered [2]: 


$$
d=\frac{m \lambda}{2 \sin \theta} \quad \Rightarrow \quad d_{h k l}=(1+\delta) \frac{\lambda}{2 \sin (\theta-\delta(\cot (\theta-\alpha)+\cot (\theta+\alpha)))} .
$$

For the temperature dependent measurements the standard Eulerian cradle, which was used for measurements at room temperature (RT), was replaced by a Huber cradle with an Anton Par high temperature chamber (HTK) or a X-ray cryostat. Compared to the Eulerian cradle, the Huber cradle also allowed a certain positioning of the sample in the incident beam, which is however reduced by the mechanics of the cradle and the geometry of the HTK. Due to the narrow windows of the HTK, a small tilt leads to a shadowing of the radiation. This provides that the maximum intensity is not measured at the correct position and the Bragg angle is not determined correctly.

Both chambers operate under vacuum and the temperature is measured by an external controller, which also manages the resistive heating. The HTK allows measurements in a temperature regime from 300 to 1210 (1500) $\mathrm{K}$ and the cryostat with a cooling by liquid helium in a regime from 20 to $500 \mathrm{~K}$. To achieve a strain free mounting of the samples and a good heat contact, these were glued by silver paste to the sample holders.

To ensure that temperature measured by the $(\mathrm{Pt} / \mathrm{RhPt})$ thermocouple $\left(T_{\mathrm{t}}\right)$ equals the temperature of the sample $(T)$, we first determined the thermal expansion of a small Si sample, directly glued on the AlN crystal, so that we can assume that both samples have the same temperature. Otherwise, a mounting of the $\mathrm{Si}$ directly on the sample holder could yield apparently wrong temperatures, because both materials might have different heat absorption and are placed at different locations in the HTK. From the comparison of the measured data with the data published by Reeber and Wang [3] we obtained a function for the calculation of the "real" temperature $T$ from $T_{\mathrm{t}}$.

\section{Results}

\subsection{Measurement of thermal expansion}

In a first set of measurements at room temperature with the Eulerian cradle the lattice constants in $c$ - and $a$-direction had been measured for both crystals. Within the results there was no difference between the single crystal and the multi-grain material. In Table I you can see the average values measured in this work and published in literature [4]. Because of the indirect measurement of the $a$-lattice constant the error for the $a$-direction is significantly bigger. An analysis of reciprocal space maps of the reflexes proves the conclusion of Bickermann et al. [1] that the spontaneously nucleated crystals are single crystals. Furthermore, we found the multi-grain material to be consisting of monocrystalline areas with a similar orientation.

As mentioned above, the usage of the Huber cradle caused some problems at the temperature dependent measurements. With a justifiable amount of time it was not possible to position the sample in such a way that the maximum intensity was measured at the correct Bragg angle. This causes a fixed offset in the measured 
TABLE I

Lattice constants at room temperature.

\begin{tabular}{l|l|c}
\hline \hline & $c$-direction $[\AA]$ & $a$-direction $[\AA]$ \\
\hline measured & $4.981 \pm 0.0004$ & $3.112 \pm 0.001$ \\
literature & $4.981 \pm 0.001$ & $3.111 \pm 0.006$
\end{tabular}

Bragg angle especially for low reflexes. Because of this, the values for lattice plane distance obtained from the 002 and the 103 reflex at room temperature were slightly bigger than those measured with the Eulerian cradle. The difference of the values obtained from the 006 and 205 reflex lies in the range of the measurement error. Due to the fact that the misalignment of the samples is constant for all temperatures, the measured Bragg angle was corrected by a constant factor for each set of data, so that the value at $300 \mathrm{~K}$ equals the value measured before at RT with the Eulerian cradle. This makes the comparison of the data sets and the simulation more comfortable, but does not alter the determination of thermal expansion coefficients.

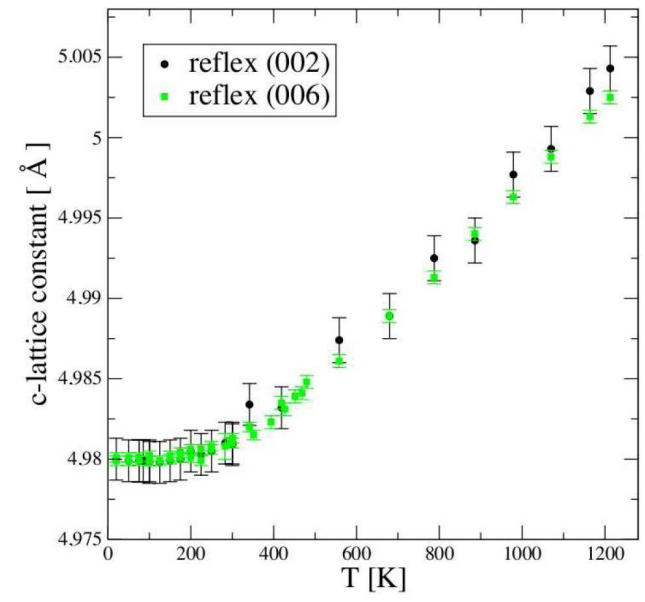

Fig. 3. Measured data for $c$-direction.

In Fig. 3 you can see the corrected data of the measured $c$-lattice constant plotted against the temperature of the sample. One can see that the data received from both reflexes agree within error bars, which were calculated from the FWHM of the X-ray reflexes. Furthermore, there was no difference between the single crystal and the multi-grain material which was investigated more detailed due to a better handling. We found the same behavior also for the $a$-direction. In Fig. 4, the data of the 006 and the average values of the 103 and the 205 reflex are plotted together with some literature data. The data published up to now was all obtained by AlN powder [5-8] or ceramics [9] and was measured either in 


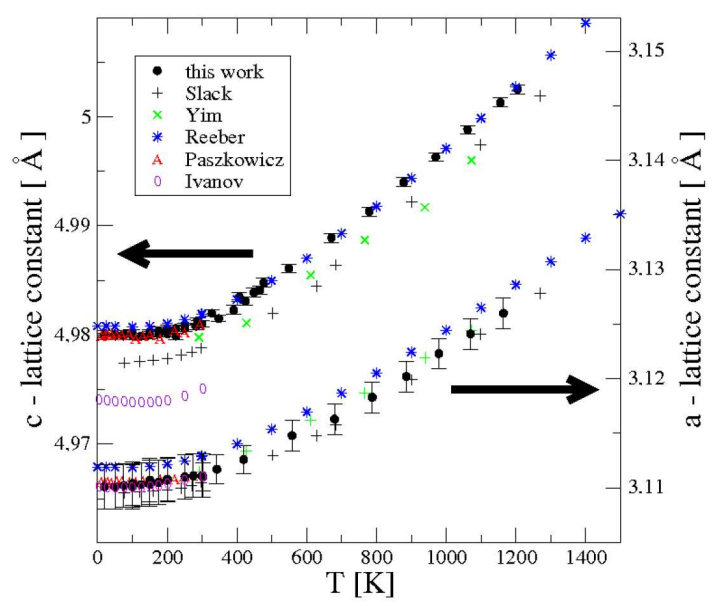

Fig. 4. Comparison of measured data with literature.

high or low temperature regime. Furthermore, the samples were investigated by different methods and differ all in chemical purity. The biggest set of data from Wang and Reeber [7] is based on the investigations of Ivanov, Yim and Slack. They simulated the data by a multi-frequency Einstein model, but did not specify the conformance with the measured data. The data presented in this study are for the first time measured on high quality crystalline samples covering a large range of temperature. In comparison to the literature data, the measured data for the $c$-lattice constant shows good conformance with the data of Reeber in the high temperature regime and at lower temperatures with the set of Paszkowicz. All the other sets, especially the data obtained from AlN ceramics by Ivanov, show lower values even at RT. The gradient at high temperature, which characterizes the high temperature expansion coefficients, fits well for all sets. For the $a$-lattice constant the measured data agrees with the literature data within error bars. Also at low temperatures the best conformance can be seen with the data of Paszkowicz. At high temperatures one can see a little lower gradient which can be caused by the indirect measurement of the lattice plane distance or by the quality of our samples. Due to the low oxygen content and the large crystal size the anisotropic behavior can be more distinctive.

\subsection{Fitting of measured data}

Assuming that the thermal expansion is proportional to the internal energy of a quantum mechanical oscillator, we used two approaches to calculate the frequency spectra of the phonons in the distribution function. One possibility is the assumption of optical phonons which build up a narrow frequency band. This is valid for high temperatures and leads to the Einstein model which is given by Eq. (2). Another possibility is the assumption of acoustic phonons which are mainly excited at lower temperatures and show a dispersion up to an upper 
limit $\omega_{\mathrm{D}}$. This leads to Eq. (3), which is called the Debye model

$$
\begin{aligned}
& U_{\mathrm{E}}(V, T)=3(s-1) G^{3} \hbar \omega_{0} f_{\mathrm{E}}\left(\frac{\Theta_{\mathrm{E}}}{T}\right) \quad \text { with } f_{\mathrm{E}}(x)=\frac{1}{\exp (x)-1}, \\
& U_{\mathrm{D}}(V, T)=3 G^{3} k_{\mathrm{B}} \Theta_{\mathrm{D}} f_{\mathrm{D}}\left(\frac{\Theta_{\mathrm{D}}}{T}\right) \quad \text { with } f_{\mathrm{D}}(x)=3 \int_{0}^{1} \frac{t^{3}}{\exp (t x)-1} \mathrm{~d} t .
\end{aligned}
$$

For the fitting of the measured data we identified the prefactors as macroscopic fit parameters, which leads to Eq. (4). Here $a_{0}$ is the lattice constant at $0 \mathrm{~K}, \alpha$ is the high-temperature thermal expansion coefficient (TEC) and $\Theta_{\mathrm{E} / \mathrm{D}}$ the characteristic Debye- or Einstein temperature

$$
a(T)=a_{0}+a_{0} \alpha \Theta_{\mathrm{E}, \mathrm{D}} f_{\mathrm{E}, \mathrm{D}}\left(\frac{\Theta}{T}\right) .
$$

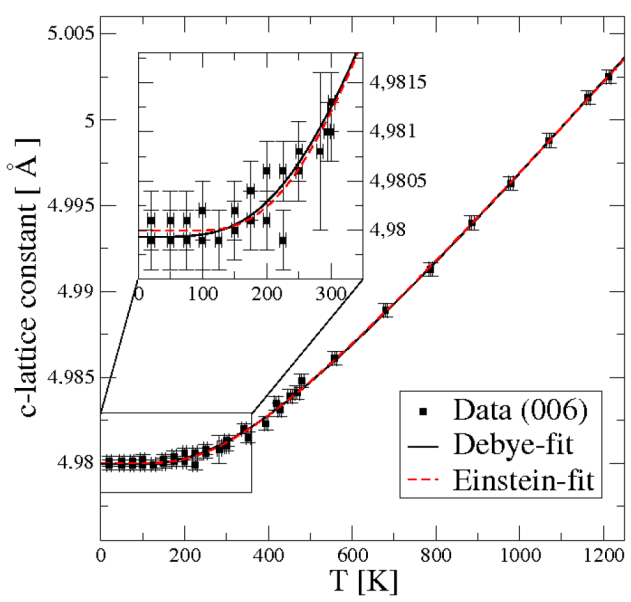

Fig. 5. Fitting of thermal expansion in $c$-lattice direction.

In Fig. 5 you can see the measured data of the 006 reflex and the fitted curves obtained by both models. One can see that both models show good conformance with the measured data over the whole range of temperature. As expected, the Debye model shows better conformance at low temperatures due to the assumption of acoustic phonons. In Table II we show the parameters received from the fitting of data shown in Fig. 4. Compared to the Debye temperature of AlN published in literature and obtained by other methods, our values are a little bit higher. The same behavior has also been found by Roder et al. [10] who investigated the thermal expansion of GaN.

A plot of the calculated TEC can be seen in Fig. 6. First, the difference between both models becomes more obvious especially at low temperatures, where the Einstein model shows a TEC of 0 below $75 \mathrm{~K}$. Second, the anisotropic behavior of AlN can be seen very well by the different graphs for $a$ - and $c$-direction. 
TABLE II

Fit parameter for thermal expansion of AlN.

\begin{tabular}{c|l|c|c|r}
\hline \hline & Model & $c_{0} / a_{0}[\AA]$ & $\alpha[1 / \mathrm{K}]$ & $\Theta_{\mathrm{E} / \mathrm{D}}[\mathrm{K}]$ \\
\hline \multirow{2}{*}{$c$} & Einstein & $4.9800 \pm 0.0001$ & $5.6 \pm 0.1 \times 10^{-6}$ & $937 \pm 25$ \\
& Debye & $4.9799 \pm 0.0001$ & $5.8 \pm 0.1 \times 10^{-6}$ & $1317 \pm 25$ \\
\hline \multirow{2}{*}{$a$} & Einstein & $3.1105 \pm 0.0002$ & $6.9 \pm 0.3 \times 10^{-6}$ & $1025 \pm 25$ \\
& Debye & $3.1104 \pm 0.0002$ & $7.1 \pm 0.3 \times 10^{-6}$ & $1455 \pm 25$
\end{tabular}

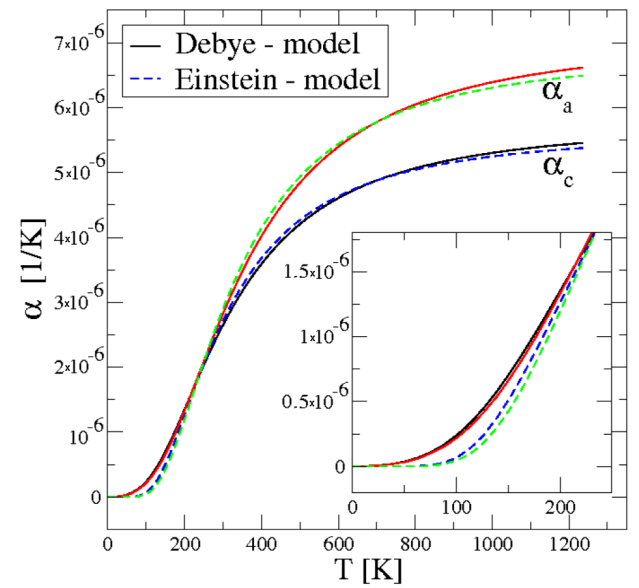

Fig. 6. Temperature dependent thermal expansion coefficient of AlN.

Furthermore, one can suggest that the TEC will become constant for very high temperatures.

\subsection{Consequence for epitaxial growth}

As mentioned before, a different thermal behavior of the materials can lead to problems for epitaxial growth. Beside the intrinsic stress, due to different lattice constants, a difference in the thermal expansion can lead to additional stress during cool down. In a system of a GaN substrate with an AlN layer, this is tensile strained due to a negative lattice mismatch. Based on our data for AlN and the data published by Roder et al. [10] for GaN we calculated the temperature dependent lattice mismatch for this system. In Fig. 7 the lattice mismatch in percent is plotted over the temperature. One can see that the mismatch passes a minimum at $700 \mathrm{~K}$, due to the lower $\alpha$ of GaN. This leads to a maximum in strain for the AlN layer, which has to be taken into account for the consideration of cracks, occurring in an originally closed layer during cool down. In this special case shown here, one has to consider that the thermal effect however plays only a secondary role, because the thermal part is still much lower than the intrinsic part of the mismatch. 


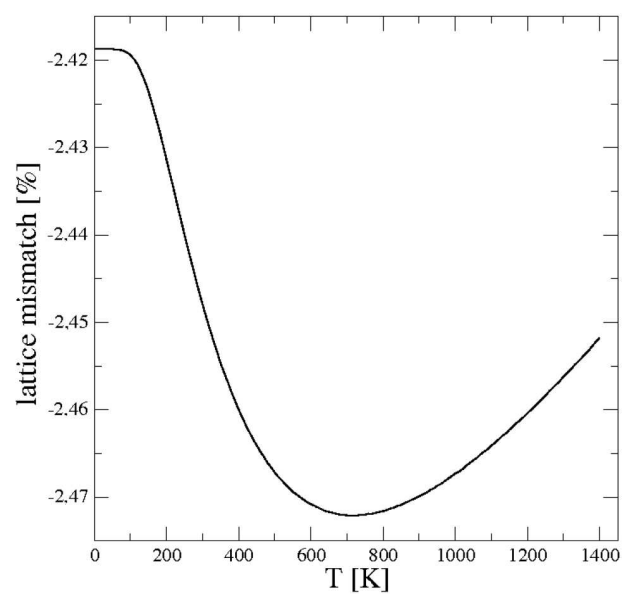

Fig. 7. Lattice mismatch in a system of GaN substrate with a AlN layer.

\section{Conclusion}

We were able to investigate AlN single crystals and polycrystalline material of high quality by high resolution XRD (HR XRD) over a range of temperature from 20 to $1210 \mathrm{~K}$. Measurements at room temperature yield $a$ - and $c$-lattice constants with high precision and good conformance with literature data. From temperature dependent measurements we received a closed set of data for the thermal expansion dependent on lattice direction. Based on this data we calculated the TEC and simulated the expansion by the Einstein- and the Debye model, which showed good conformance with the measured data. Furthermore, we showed that the thermal expansion leads to a maximum in strain of epitaxial AlN layers on GaN substrate during cool down from growth temperature, whereas the thermal part is small compared to the intrinsic strain.

\section{References}

[1] M. Bickermann, B.M. Epelbaum, A. Winnacker, J. Cryst. Growth 269, 432 (2004).

[2] P.F. Fewster, N.L. Andrew, J. Appl. Crystallogr. 28, 451 (1995).

[3] R.R. Reeber, K. Wang, Mater. Chem. Phys. 46, 259 (1996).

[4] Landolt-Börnstein, Group IV Elements, IV-VI and III-V Compounds, Vol. 41, Springer-Verlag, Berlin 2001, p. 370.

[5] G.A. Slack, S.F. Bartram, J. Appl. Phys. 46, 8998 (1975).

[6] W.M. Yim, R.J. Paff, J. Appl. Phys. 45, 14561457 (1974).

[7] K. Wang, R.R. Reeber, Mater. Res. Soc. Symp. Proc. 482, 863868 (1998).

[8] W. Paszkowicz, M. Knapp, S. Podsiadlo, G. Kamler, J.B. Pelka, Acta Phys. Pol. A 101, 781 (2002).

[9] S.N. Ivanov, P.A. Popov, B.I. Kornev, Phys. Solid State 39, 8183 (1997).

[10] C. Roder, S. Einfeld, S. Figge, D. Hommel, Phys. Rev. B 72, 085218 (2005). 